\title{
Phonon Scattering in Quantitative High-Resolution Electron Microscopy - effects, problems and approaches
}

\author{
Zhong Lin (ZL) Wang \\ School of Materials Science and Engineering, Georgia Institute of Technology, Atlanta, GA \\ 30332-0245 USA \\ * E-mail: zhong.wang@mse.gatech.edu
}

In quantitative high-resolution transmission electron microscopy, the theoretically calculated images usually give better contrast than the experimentally observed ones although all of the factors have been accounted for. This discrepancy is suggested due to thermal diffusely scattered (TDS) electrons [1], which were not included in the image calculation. The contribution from TDS electrons is especially important if the image resolution is approaching $0.1 \mathrm{~nm}$ and beyond with the introduction of Cs corrected microscopes. It is shown that the contribution of the TDS electrons to the image is of the same order as the cross interference terms for the Bragg reflected beams in the dark-field high-resolution TEM imaging. A more rigorous multislice theory has been developed to account for this effect [2].

In electron scattering, most of the existing dynamical theories have been developed under the first order diffuse scattering approximation, thus, they are restricted to cases where the lattice distortion is small. A formal dynamical theory is presented for calculating diffuse scattering with the inclusion of multiple diffuse scattering $[3,4,5]$. By inclusion of a complex potential in dynamical calculation, a rigorous proof is given to show that the high order diffuse scattering are fully recovered in the calculations using the equation derived under the distorted wave Born approximation, and more importantly, the statistical time and structure averages over the distorted crystal lattices are evaluated analytically prior numerical calculation. This conclusion establishes the basis for expanding the applications of the existing theories.

The "frozen lattice" model is a semi-classical approach for calculating electron diffuse scattering in crystals arisen from thermal vibration of crystal atoms. This quasi-elastic scattering approach is, however, concerned since its equivalence with the incoherent phonon excitation model is not yet established. Based on a rigorous quantum mechanical phonon excitation theory, we have proved that an identical result would be obtained using the frozen lattice model and the formal phonon excitation model if, 1) the incoherence between different orders of thermal diffuse scattering is considered in the frozen lattice model calculation, and 2) the specimen thickness and the mean-freepath length for phonon excitation both are smaller than the distance traveled by the electron within the life-time of the phonon ( $5 \mu \mathrm{m}$ for $100 \mathrm{kV}$ electrons) [6]. Condition 2 ) is usually absolutely satisfied and condition 1) can be precisely accounted for in the calculation with the introduction of the mixed dynamic form factor $\mathbf{S}\left(\mathbf{Q}, \mathbf{Q}^{\prime}\right)$ [4]. The conclusion holds for each and all of the orders of diffuse scattering, thus, the quantum mechanical basis of the frozen lattice model is established, confirming the validity, reliability and accuracy of using the frozen lattice model in quantitative dynamical electron diffraction and imaging calculations.

[1] Z.L. Wang, "Phonon Scattering, How Does it Affect the Image Contrast in High-Resolution TEM?" Phil. Mag. B, 79 (1999), p. 37-48.

[2] Z.L. Wang, "An Optical Potential A roach to Incoherent Multiple Thermal Diffuse Scattering in Quantitative HRTEM,” Ultramicroscopy, 74 (1998), p. 7-26. 
[3] Z.L. Wang, "Statistical Multiple Diffuse Scattering in a Distorted Crystal System - An Exact Theory," Phil. Mag. B 74 (1996), p. 733-749.

[4] Z.L. Wang, "Electron Statistical Dynamical Diffuse Scattering in Crystals Containing ShortRange Order Point Defects," Acta Cryst. A52 (1996), p. 717-729.

[5] Z.L. Wang, "Green's Function for Electron Scattering and its Applications in Low Voltage Point-Projection Microscopy and Optical Potential," Phil. Mag. B, 77 (1998), p. 787-803.

[6] Z.L. Wang, "The 'Frozen Lattice' A roach for Incoherent Phonon Excitations in Electron Scattering, How Accurate is it?" Acta Cryst. A, 54 (1998), p. 468-480.

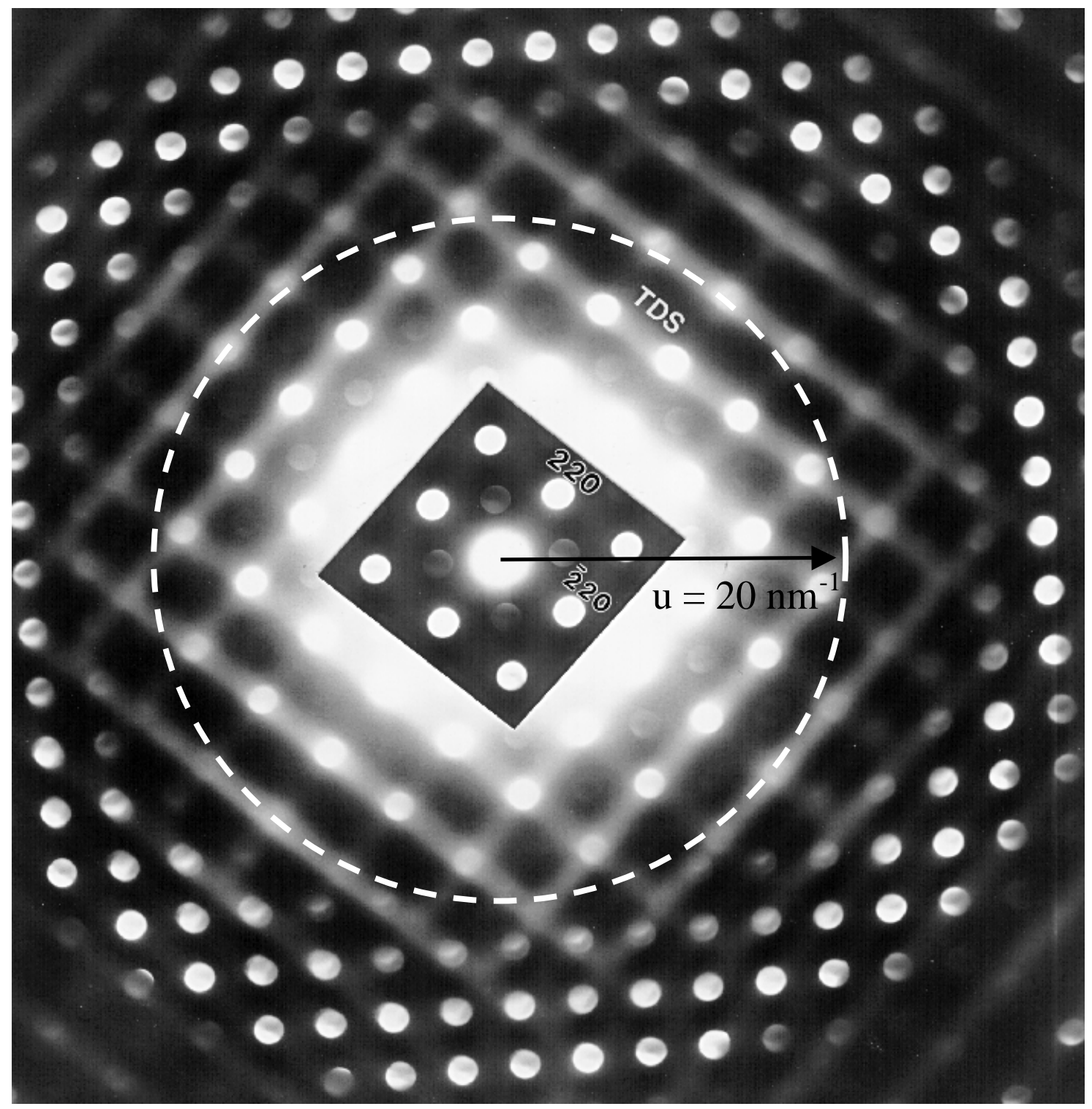

FIG. 1 Electron diffraction pattern of GaAs showing the streaks due to thermal diffuse scattering at room temperature at high scattering angles. The TDS streaks will strongly affect the contrast of electron image at the atom sites when the image resolution approaches $0.1 \mathrm{~nm}$ and beyond. 